This document is published in:

Cybernetics and Systems: An International Journal (2012), 43(3), 163-180.

DOI: 10.1080/01969722.2012.659977

(c) 2012 Taylor \& Francis Group, LLC 


\title{
TEO: FULL-SIZE HUMANOID ROBOT DESIGN POWERED BY A FUEL CELL SYSTEM
}

(Short version of the title: TEO robot design powered by a fuel cell system)

\author{
S. MARTÍNEZ*, C.A. MONJE*, A. JARDÓN*, P. PIERRO*, C. BALAGUER*, D. MUÑOZ** \\ *System Engineering and Automation Department, University Carlos III of Madrid, \\ Avenida Universidad 30, 28911 Leganés, Madrid, Spain \\ \{scasa,cmonje,ajardon,ppierro,balaguer\}@ing.uc3.es \\ **Hynergreen Technologies, S.A., \\ Avenida de la Buhaira 2, 41018 Sevilla, Spain \\ delia.munoz@hynergreen.abengoa.com
}

This paper deals with the design of the full size humanoid robot TEO, an improved version of its predecessor RH-1. The whole platform is conceived under the premise of high efficiency in terms of energy consumption and optimization. We will focus mainly on the electromechanical structure of the lower part of the prototype, which is the main component demanding energy during motion. The dimensions and weight of the robotic platform, together with its link configuration and rigidity, will be optimized. Experimental results are presented to show the validity of the design.

Keywords: Humanoid robot design; energy consumption; lower body improvement.

\section{INTRODUCTION}

The humanoid robotics group RoboticsLab, at the University Carlos III of Madrid, has been working for many years in the RH-project, a robust and open humanoid platform for research on biped walking, balancing control, sensor fusion, human-robot interaction (HRI) for collaborative task, and other related issues.

RH-1 is an anthropomorphic robot with 21 degrees of freedom, a height of $1.5 \mathrm{~m}$, and a weight of about 
$50 \mathrm{~kg}$. The main research objectives of this platform have been the stability of the robot (Kaynov et al. 2009) and the gait generation (Pardos and Balaguer 2005; Arbulú and Balaguer 2007), though other research activities have focused on human-robot interaction (Staroverov, Ricardo Martinez, et al. 2007; Staroverov, Marcos, et al. 2007) and collaboration (Pierro, Monje, and Balaguer 2008). In fact, these two first versions aim at studying stable walking and do not consider upper part movements related to physical collaboration.

Though stable walking has been achieved in this platform, several aspects have to be improved. As a main problem, the mechanical structure of this prototype is not very robust and presents limitations such as the high flexibility of the whole body and the joints looseness, which not only complicates the stability control but also limits the range of applications to be performed with the robots, not to mention the increase of energy consumption.

However, the most relevant humanoid platforms came out as a step by step improvement of different versions, such as Asimo (Sakagami et al. 2002), HRP-3 (Kaneko et al. 2008), Hubo (Park et al. 2005), or Wabian-2 (Ogura et al. 2006). Other relevant platforms were specifically designed for peculiar applications: Jonnie (Pfeiffer, Loffler, and Gienger 2002) was initially designed for fast-walking, and i-Cub (Metta et al. 2008) for research on embodied cognition. In this line, the new prototype TEO appears as an improved version of its predecessor RH-1.

TEO (Pabon et al. 2009; Carlos Martinez et al. 2009) addresses challenges in the fields of motion, safety, energy efficiency, and power autonomy performance. The mechatronics of this platform is inspired by the human natural and adaptive locomotion, and its design is oriented to achieve human physical capacities and performances.

An important issue is that the whole platform is conceived under the premise of high efficiency in terms 
of energy consumption and optimization. In general terms, the existing prototypes of humanoid robots are heavy to carry and have limited energy capacity. A Honda humanoid robot can walk for only 30 minutes with a battery pack it carries on the back. Energy is one of the most important challenge for mobile robots in general. For instance, (Rybski et al. 2000) show that power consumption is one of the major issues in their robot design.

There are several components in a humanoid robot that demand energy consumption, such as motors, sensors, microcontrollers, and embedded computers. However, one of the most relevant terms in the whole energy balance is motion power. DC motors transform direct current into mechanical energy to drive the robots. The electromechanical structure is therefore the main component demanding energy. The dimensions and weight of the robotic platform, together with its link configuration and rigidity, will affect the final energy consumption. We will focus mainly on these electromechanical aspects when designing the new prototype TEO, and more specifically its lower part, as will be detailed along the paper. Besides, other aspects, such as motion planning, will be considered to reduce motion power.

The rest of the paper is organized as follows. Section 2 shows some previous experiences with RH-1, stating the improvements required for TEO design. In Section 3 a methodology is established in order to perform the new design steps successfully. Section 4 presents three case studies focusing on each of the main components of the lower part of the robot: the ankle, the tibia-thigh set, and the hip. Simulation and experimental results of these designs are given. Finally, Section 5 presents some conclusions and future works.

\section{PREVIOUS EXPERIENCES WITH RH-1}

As stated before, the RH-1 version of the robot was realized from scratch, and even if it has been possible to perform different walking and stability strategies, it presented several limitations. 
However, such platform had its strength in the following points:

- Structural analysis: In order to perform an accurate analysis, support of external forces, links geometries, and material properties were considered, including reaction forces and material stresses.

- Design of the mechanical part: the mechanical design used the human structure as reference.

- Inverse dynamics analysis: this tool has been reused for future calculations in TEO.

- Behavior of the structure during the movements: once the platform was designed, it was successfully tested under stress conditions due to continuous walking movements.

Nevertheless, RH-1 robot presented great limitations which did not allow a correct motion performance in terms of mechanical robustness (high joint looseness), stability, and energy consumption (necessity to be connected to the electrical net since battery could not supply the required energy for more than a while), not to mention the realization of high-level tasks such as manipulation, complex gait generation, or complex human-robot interaction.

One of the main problems of the design was that the structure was oversized, which implied the overweight of the prototype. This leaded not only to mechanical looseness of the body but also to devote more control efforts to guarantee the stability of the system, computationally and mechanically speaking.

In the analysis of the mechanical structure, only the strength analysis was considered. A precise study of displacement methods - that make use of the components stiffness relations for computing forces in the structure - was omitted. The design constraint was simply a large factor of safety. As a result, the structure resulted in wide backlashes, which were undesirable for the high precision required.

In addition, the upper part of the robot presented several limitations. In fact, the workspace for both legs 
and arms needed to be extended. In particular, it was concluded that two DOF had to be added to each arm, and two more to the weist of the robot, resulting in a wider workspace and higher manipulability in the different configurations. This new configuration has been set in the new TEO, with a total of 26 DOF.

Finally, it is important to stress the difference between the structure of these previous prototypes and the new one. RH-1 presented a structure based on the human one: the robot had a real skeleton to which all the electronic components were attached. This caused several problems when new electronic components needed to be added, due to the lack of space.

The new structure of TEO is based on a box concept: the robot structure itself is a container in which all the electronic components are inserted. More details will be given in the following sections of the paper. A scheme summarizing the improvements proposed of the electromechanical structure is presented in Fig. 1.

Figure 1. Improvements of the electromechanical structure.

\section{DESIGN PROPOSALS FOR THE LOWER BODY IMPROVEMENT}

Once the main limitations of the prototype are studied, a design methodology is needed to carry out the improvements successfully, focusing on the lower part of the robot (though the same flow is applied to the upper part). We will follow the scheme from Fig. 2.

Figure 2. Development process flow.

As can be seen in this figure, the process is divided into three stages:

1. The design stage. The concept for the lower part is stablished and a first mechanical structure is 
proposed and modelled. The main result of this process is the 3D CAD model of the robot parts and assemblies.

2. The simulation stage. The aim of this stage is to obtain numerical data that allow the optimization of the structural parts of the robot, the selection of the appropriate movement chain (motor+reduction), and the estimation of the energy consumption. This process is divided into two parts: a) a task simulation step, in which torques, work angles, and other performance parameters are calculated; b) a finite element (FEM) study of each structural component. There is a feedback between both simulations steps in order to make an optimization of the parts and systems through several iterations.

3. The manufacturing stage. Once design, simulation, and optimization processes have finished, the manufacturing of the structural parts and their assembly is the final step.

Taking into account a more realistic evolution of the efforts that the lower part of the robot suffers during the walking of TEO, a dynamic simulation of the multi-body model of the robot has to be performed in order to obtain the real forces and loads applied to each piece. This way, these pieces can be redesigned so that they can support those efforts with the minimum weight.

Therefore, the first step is to obtain the multi-body model of the lower part, that allows the study of the dynamics of the pieces taking into account the interaction between them and the reaction forces. Fig. 3 presents the resulting model, showing the situation of the different centers of masses and the corresponding links between elements. All the calculations are made using ABAQUS/Standard, a commercial software specially devoted to the advanced calculus of static and dynamic efforts over elements.

Figure 3. Multi-body model of the lower part of humanoid robot TEO. 
The floor has been characterized as a horizontal infinitely rigid surface and the contact between this surface and the inferior surface of the foot has been declared. All pieces are of aluminium, and the sole of the foot is made of a generic type of polymere. To model the behaviour of both the aluminium and the polymer, the characteristics shown in Table 1 have been considered.

Table 1. Characteristics of aluminium and polymer.

\begin{tabular}{|c|c|c|c|}
\cline { 2 - 4 } \multicolumn{1}{c|}{} & Young Module & Poisson Module & Density \\
\hline Aluminium & $\mathrm{E}=70 \mathrm{GPa}$ & $v=0.4$ & $2810 \mathrm{~kg} / \mathrm{m}^{3}$ \\
\hline Polymer & $\mathrm{E}=250 \mathrm{GPa}$ & $v=0.05$ & $1800 \mathrm{~kg} / \mathrm{m}^{3}$ \\
\hline
\end{tabular}

It is necessary to clarify that a null density has been given to the elements of the robot, since the masses have been considered as concentrated in the centers of gravity of each element.

Under these conditions, a nominal trajectory is simulated (see Fig. 4), leading to the necessary conclusions for the redesign of the low part according to the forces and moments obtained for each element and their directions.

Figure 4. Global model walking.

\section{CASE STUDIES}

This section addresses the design of the three main components of the lower part of the humanoid robot TEO. The improvements of the ankle, the tibia-thigh set, and the hip are presented and discussed as three different case studies, including simulation and experimental results regarding their specific and more restrictive requirements of design. 


\section{IV.I CASE STUDY 1: THE ANKLE}

During the walking motion, the ankle is the most critical joint due to the forces and torques it has to bear. On one hand, this joint must support the weight of the robot during the single support phase in a locomotion task, in which only one foot has contact with the ground. On the other hand, the ankle is the nearest joint of the robot kinematic chain to the ground. When the flying foot lands, the force of the impact is transmitted firstly to this joint. These two effects have to be taken into account in the design of the ankle.

As its structure must have a compact size, a very little backslash in this joint causes balance loss or increases instability. The mechanical design of this joint should avoid this problem, assuring the stability while the robot is walking. Besides, avoiding backslash helps the control system to perform precise walking movements.

In a first stage of design, the 3D CAD model of the ankle parts and assemblies is obtained, as shown in Fig. 5.

Figure 5. Previous design of robot ankles.

Then, the simulation stage is addressed, as explained in Section 3. It is evident that the humanoid robot is a very dynamical system and the loads on its parts vary depending on the task executed. The robot walking task has been selected for this simulation stage, since it is one of the most demanding. Using Matlab Simmechanics ${ }^{\mathrm{TM}}$, the walking task has been simulated obtaining data regarding joints torques, angular velocities, and accelerations. Fig. 6 shows the results of the right ankle during the two phases that one foot goes through in a walking period: on the ground (supporting phase) and flying.

Figure 6. Frontal and sagittal angles and torques of the right ankle. 
As can be observed in Fig. 6, the torque depends on the supporting phase during the walking task. The moments in which the torques are bigger correspond to the end of each support phase. The analysis of the torques is useful in the selection of the transmission chain and the motors of the joints. The RMS value determines the nominal torque that the motor must support during a walking period. On the other hand, the peak torque determines the minimun stall torque that the motor must supply in order to start the movement. The summary of the walking simulation results is shown in Table 2 .

Table 2. Summary of results from Matlab Simmechanics simulations.

\begin{tabular}{|c|c|c|c|c|}
\cline { 2 - 5 } \multicolumn{1}{c|}{} & Joint Torque & Reduction & Motor Torque & Selected Motor \\
\hline Sagittal Joint & $\begin{array}{l}\text { RMS=3.4 Nm } \\
\text { Max=40.6 Nm }\end{array}$ & 235.2 & $\begin{array}{l}\text { RMS=14.4 mNm } \\
\text { Max=173 mNm }\end{array}$ & Maxon Brushless EC45 Flat 251601 \\
\hline Frontal Joint & $\begin{array}{l}\mathrm{RMS}=27.7 \mathrm{Nm} \\
\mathrm{Max}=85 \mathrm{Nm}\end{array}$ & 320 & $\begin{array}{l}\mathrm{RMS}=86.6 \mathrm{mNm} \\
\mathrm{Max}=265 \mathrm{mNm}\end{array}$ & Maxon Brushless EC45 Flat 339287 \\
\hline
\end{tabular}

Other simulation tools have been used to test other features during the walking action. For example, one important aspect of the task performance is the stability. Using OpenHRP (see Fig. 7), a simulation software for humanoid platforms, this feature has been tested and gaits have been modified and improved in order to guarantee the stability of the structure during the walking action.

Figure 7. OpenHRP simulations.

After these simulations, and using ABAQUS/Standard numeric simulation software, the main parts of the ankle that have been analyzed and optimized are the fork and the so called cross. These parts allow the ankle to turn in the frontal and sagittal planes and support the whole robot weight during the single support phase. These parts are joined to the leg by means of the transmission pack, a mechanical structure which contains the Harmonic Drive components, assembled with steel screws. The main goal of this optimization loop has been the reduction of weight without loosing strenght features. 
Fig. 8 and Fig. 9 show the results of the FEM simulation of the ankle. The fork and cross are made of Aluminium 7075 alloy. For the study, the elastic limit $\left(\mathrm{R}_{\mathrm{P} 0.2}=110 \mathrm{Mpa}\right)$ has been considered instead of the fracture limit $(\mathrm{Rm}=160 \mathrm{MPa})$, since permanent deformations of the parts are not allowed and $\mathrm{R}_{\mathrm{P}}$ is more restrictive. The resulting physical features of the model have been feedbacked later to the Matlab Simmechanic model to perform a new iteration of simulations.

Figure 8. FEM study of the cross part.

Figure 9. FEM study of the fork part.

Once the design, simulation, and optimization processes have finished, the manufacturing of the structural parts and their assembly is carried out, as shown in Fig. 10.

Figure 10. Assembled ankle: cross and fork manufactured parts.

\section{IV.II CASE STUDY 2: THE TIBIA-THIGH SET}

Under the premise that the new prototype must fulfil strict requirements of weight, a redesign of the tibia-thigh set has also been carried out. The initial design was very conservative when considering security criteria and forces applied over this set during the walking action in different conditions.

To this respect, it is important to remark that RH-1 presented a structure based on the human one: the robot had a real skeleton to which all the electronic components were attached. This caused several problems when new electronic components needed to be added, due to the lack of space.

The new structure of TEO is based on a box concept: the robot structure itself is a container in which all 
the electronic components are inserted. This kind of structure involves all electronic and mechanical subsystems, protecting them, and provides more stiffness and robustness in general, specially in case of a nasty fall.

Taking this into account, a procedure similar to the one explained in the previous case study is followed. Based on this process results, the redesign of the different pieces is performed taking as input conditions (forces, moments) those given by the simulations. After 12 iterations, the final model for the tibia is the one in Figure 11, with the maximum possible reduction of weight.

Figure 11. Tibia model R24. Weight: 917g; Maximum Von Mises tension: $2.32 \mathrm{e}^{8} \mathrm{~N} / \mathrm{m}^{2}$.

The final manufactured tibia and thigh are presented in Fig. 12.

Figure 12. Manufactured tibia (up) and thigh (down).

\section{IV.III CASE STUDY 3: THE HIP}

The last significant improvement regarding the mechanical design of the lower part is the replacement of the cantilever supporting structure of RH-1 by a planar design in TEO. Though it implies more design efforts, the robot stiffness is considerably improved, avoiding the flexural problems not really solved in RH-1.

Based on required trajectories, dynamic simulations has been performed in order to optimally define the geometrical shape of the hip and its thickness (both fork and cross parts, as in the ankle), and to avoid internal tension concentration points. Fig. 13 shows the FEM analysis performed to the fork part of the hip. The FEM study presented for the cross part of the ankle validates also the fork part of the hip, since in this case the mechanical efforts are lower. Special considerations of required speed and torque of the 
additional motors have been taken in account in order to keep the power requirements lower enough.

Figure 13. Hip cross part model of the humanoid robot TEO.

A picture of the manufactured parts of the hip is shown in Fig. 14.

Figure 14. Manufactured hip cross part (left) and hip fork part (right).

Acting similarly with the rest of elements of the legs, the final result is a weight reduction of $356 \mathrm{~g}$ per leg, which implies a global weight reduction of $712 \mathrm{~g}$ in the lower body of the humanoid robot. This reduction is still conservative and a security coefficient is guaranteed to ensure the correct performance of the structure in the worst walking conditions.

\section{CONSIDERATIONS ON THE ENERGY SYSTEM DESIGN}

After analyzing TEO's power requirements, the average continuous power required due to the electronic on-board system is about $150 \mathrm{~W}$, that is, when there is no movement at any extremity but all the drives are enable and holding the robot position. At typical walking speeds, the power requirements are shown in Table 3. This global consumption must be taken into account when considering the type of energy source to be used in our platform.

Table 3. Summary of typical power requirements of the lower train.

\begin{tabular}{|l|c|c|}
\cline { 2 - 3 } \multicolumn{1}{c|}{} & $\begin{array}{c}\text { Load Per Joint } \\
(\mathrm{Nm})\end{array}$ & $\begin{array}{c}\text { Power } \\
(\mathrm{W})\end{array}$ \\
\hline Ankle Sagittal Joints (x2) & $18.7 \mathrm{RMS}$ & 56.6 \\
\cline { 2 - 3 } & $40.6 \mathrm{Max}$. & 123.8 \\
\hline Ankle Frontal Joints (x2) & $27.7 \mathrm{RMS}$ & 30.6 \\
\cline { 2 - 3 } & $85.0 \mathrm{Max}$. & 94.3 \\
\hline Knee Joints (x2) & $25.4 \mathrm{RMS}$ & 31.3 \\
\cline { 2 - 3 } & $72.0 \mathrm{Max}$. & 90 \\
\hline
\end{tabular}




\begin{tabular}{|l|c|c|}
\hline Hip Frontal Joints (x2) & $10.2 \mathrm{RMS}$ & 18 \\
\cline { 2 - 3 } & $26.0 \mathrm{Max}$. & 46.8 \\
\hline Hip Sagittal Joints (x2) & $19.1 \mathrm{RMS}$ & 36 \\
\cline { 2 - 3 } & $84.0 \mathrm{Max}$. & 158.4 \\
\hline \multirow{3}{*}{ Hip Axial Joints (x2) } & $3.2 \mathrm{RMS}$ & 7.2 \\
\cline { 2 - 3 } & $7.3 \mathrm{Max}$. & 18 \\
\hline \multirow{5}{*}{} & Total $\mathrm{P}_{\mathrm{RMS}}(\mathrm{W})$ & 360 \\
\cline { 2 - 3 } & Total $\mathrm{P}_{\max }(\mathrm{W})$ & 1062 \\
\cline { 2 - 2 }
\end{tabular}

As a first approach, we have considered a fuel cell (Hoogers 2003) as the energy source for our TEO platform, in order to avoid the energy problems previously experienced with RH-1. The key feature of small fuel cells to be used as battery replacements is the running time without recharging. Obviously, by definition, the size and weight are also important. Power units with either significantly higher power densities or larger energy storage capacities than other existing batteries may find applications in portable computers, communication, and transmission devices, as it is the case.

The fuel cell electric generator system proposed for TEO is composed of five elements: hydrogen storage system, fuel cell system, balance of plant (BoP), control system, and power conditioning system. A scheme of the whole system is presented in Fig. 15. The energy source of the fuel cell, the hydrogen, will be stored in the fuel storage system. It will consist of several high pressure hydrogen tanks that will be installed in the waist of the robot. The fuel cell system is based on Proton Exchange Membrane fuel cell (PEM). This fuel cell takes advantage on the energy content in hydrogen to generate the electrical power. The BoP refers to supporting and/or auxiliary components (regulators and valves) that help the hydrogen to reach the fuel cell in appropriate conditions.

Figure 15. Hybrid fuel cell simplified scheme.

The ideal power requirement for the hydrogen cell is a continuous current discharge. Obviously, the robot actuators require non homogeneous performance curves, which means that the peak and continuous (rms) 
power requirements must be addressed. Therefore, a converter is necessary to smooth the power demand at cell side and it is responsible of the power conditioning system. The role of this system is crucial to optimize energy transfer during task cycles. In this sense, the auxiliary rechargeable battery must both add power peak capabilities and also store energy when the generated power is higher than the total consumption. Finally, the control system manages the operation of the fuel cell engine, collects operational data and controls the possible alarms.

Taking into account the configuration and properties of the whole fuel cell generator, the space requirements for the installation of this system must be evaluated. Fig. 16 shows the allocation of the elements of the power system, to be placed at the back of the robot (left), and the adaptation of the real fuel cell to the robot (right). Other additional volumes along the robot structure can be used in case of necessity. The challenge here is to adjust the power capability of the whole hybrid system optimizing at the same time: volume, weight, power transferences from/to the energy system and its allocation inside the robot body.

Figure 16. Volume and allocation of the power system

We are currently working on the specific requirements regarding the whole fuel system installation, and also studying the dynamic model of the cell in order to control its working point according to the power demand from the different taks being performed.

\section{CONCLUSIONS AND FUTURE WORKS}

Humanoid robot TEO is the successful result of several years of research of the humanoid robotics group RoboticsLab at University Carlos III of Madrid. The design of this full size humanoid robotic platform has been presented throughout the paper. The methodology in the electromechanical design of a robotic platform is one of the critical point in this work. In fact, the new version has been adapted to the planned 
constraints related to the tasks to be realized.

The previous version RH-1 presented strong limitations in the energy efficiency. The proposed methodology focuses on the energy consumption reduction. It has been shown how it is possible to increase the energy efficiency through an optimal mechanical design of the structure and a correct choice of the electrical components.

RH-1 robot presented several additional weak points. The first critical point was the high flexibility of the structure, which complicated the control and the stabilization of the robot. The new design has been oriented to overcome such drawback.

In particular the paper focuses especially in the design of the lower part of the robot, which is the most critical from the energy point of view, and also from the control point of view. Great attention has been paid to the design of the ankle joint. The improvements with respect to RH-1 robot ankle are related to the increment of the joint motion range and its payload. Thus, the axis joint has a wide section and it is made only in one piece. Moreover, this axis aligns permanently the three pieces of the Harmonic Drive with three bearings, ensuring the load transmission along the mechanical chain. Besides, motion range is increased in sagittal plane. This improvement allows longer steps and the center of gravity height is lowered with respect to RH-1 humanoid robot. With all these advantages, faster and more stable motion will be obtained.

Another great limitation of the previous version was the reduced workspace of the upper part, which is now the main point of study of the research group. In particular, the aim is to provide the upper part with wider workspace and more manipulability.

The optimization of the fuel cell is also a current research activity of the humanoid robotics group 
RoboticsLab.

\section{ACKNOWLEDGEMENTS}

The research leading to these results has received funding from the RoboCity2030-II-CM project (S2009/DPI-1559), funded by Programas de Actividades I+D en la Comunidad de Madrid and cofunded by Structural Funds of the EU.

\section{REFERENCES}

Arbulú, Mario, and Carlos Balaguer. 2007. Real-time gait planning for Rh-1 humanoid robot, using Local Axis Gait algorithm. In 7th IEEE-RAS International Conference on Humanoid Robots, 6:563-568. Pittsburgh: IEEE. doi:10.1109/ICHR.2007.4813927. http://ieeexplore.ieee.org/lpdocs/epic03/wrapper.htm?arnumber=4813927.

Hoogers, Gregor. 2003. Handbook of Fuel Cell Technology. Ed. Gregor Hoogers. New York. CRC Press. http://www.loc.gov/catdir/toc/fy035/2002067086.html.

Kaneko, Kenji, Kensuke Harada, Fumio Kanehiro, G Miyamori, and K Akachi. 2008. Humanoid robot HRP-3. In Intelligent Robots and Systems 2008 IROS 2008 IEEERSJ International Conference on, 2471-2478. doi:10.1109/IROS.2008.4650604.

Kaynov, Dimitry, Philippe Souères, Paolo Pierro, and Carlos Balaguer. 2009. A practical decoupled stabilizer for joint-position controlled humanoid robots. In IEEE-RSJ International Conference on Intelligent Robots and Systems, 3392-3397. IEEE. doi:10.1109/IROS.2009.5354431. http://ieeexplore.ieee.org/lpdocs/epic03/wrapper.htm?arnumber=5354431.

Martinez, Carlos, Paolo Pierro, Santiago Martinez, Luis Pabon, Mario Arbulú, and Carlos Balaguer. 2009. RH-2 an Upgraded Full-size Humanoid Platform. In Proceedings of the Twelfth International Conference on Climbing and Walking Robots and the Support Technologies for Mobile Machines, 
ed. O Tosun, H L Akin, M O Tokhi, and G S Virk, 471-478. Istanbul.

doi:10.1142/9789814291279_0058.

Metta, Giorgio, Giulio Sandini, David Vernon, Lorenzo Natale, and Francesco Nori. 2008. "The iCub humanoid robot: an open platform for research in embodied cognition.” Ed. Raj Madhavan and Elena Messina. Electronics: 50-56. doi:10.1145/1774674.1774683.

http://portal.acm.org/citation.cfm?id=1774683.

Ogura, Yu, Hiroyuki Aikawa, Kazushi Shimomura, Hideki Kondo, Akitoshi Morishima, Hun-ok Lim, and Atsuo Takanishi. 2006. Development of a new humanoid robot WABIAN-2. In Robotics and Automation, 2006. ICRA 2006. Proceedings 2006 IEEE International Conference on, 76-81. IEEE. doi:10.1109/ROBOT.2006.1641164. http://ieeexplore.ieee.org/lpdocs/epic03/wrapper.htm?arnumber=1641164.

Pabon, Luis, Carlos Martinez, Jorge Villagra, and Carlos Balaguer. 2009. Mechatronic design and control of a critical biped robot joint. In 2009 IEEE International Conference on Mechatronics, 1-6. IEEE. doi:10.1109/ICMECH.2009.4957206. http://ieeexplore.ieee.org/lpdocs/epic03/wrapper.htm?arnumber=4957206.

Pardos, Jose Manuel, and Carlos Balaguer. 2005. Humanoid Robot Kinematics Modeling Using Lie Groups. In Climbing and Walking Robots, 569-575. Springer. http://roboticslab.uc3m.es/publications/ICRA05_a773_v1.pdf.

Park, Ill-Woo, Jung-Yup Kim, Jungho Lee, and Jun-Ho Oh. 2005. Mechanical Design of Humanoid Robot Platform KHR-3 (KAIST Humanoid Robot-3: HUBO). In Proceedings of 2005 5th IEEE-RAS International Conference on Humanoid Robots, 321-326. Tsukuba: IEEE. doi:10.1109/ICHR.2005.1573587. 
Pfeiffer, Friedrich, Klaus Loffler, and Michael Gienger. 2002. The concept of jogging JOHNNIE. In Robotics and Automation, 2002. Proceedings. ICRA 'O2. IEEE International Conference on, 3:3129-3135. Washington: IEEE. doi:10.1109/ROBOT.2002.1013708. http://www.csa.com/partners/viewrecord.php?requester=gs\&amp;collection=TRD\&amp;recid=6275 $11 \mathrm{CI}$.

Pierro, Paolo, Concepcion Monje, and Carlos Balaguer. 2008. Modelling and Control of the Humanoid Robot RH-1 for Collaborative Tasks. In Humanoid Robots, 2008. Humanoids 2008. 8th IEEE-RAS International Conference on, ed. IEEE, 125-131. Daejon. doi:10.1109/ICHR.2008.4755942.

Rybski, Paul E., Nikolaos P. Papanikolopoulos, Sascha A. Stoeter, Donald G. Krantz, Kemal B. Yesin, Maria Gini, Richard Voyles, Dean F. Hougen, Brad Nelson, and Michael D. Erickson. 2000. "Enlisting rangers and scouts for reconnaissance and surveillance." IEEE Robotics \& Automation Magazine 7 (4): 14-24. doi:10.1109/100.894029.

Sakagami, Yoshiaki, Ryujin Watanabe, Chiaki Aoyama, Shinichi Matsunaga, Nobuo Higaki, and Kikuo Fujimura. 2002. The intelligent ASIMO: system overview and integration. In Intelligent Robots and Systems, 2002. IEEE/RSJ International Conference on, 3:2478-2483. Lausanne: IEEE. doi:10.1109/IRDS.2002.1041641. http://ieeexplore.ieee.org/lpdocs/epic03/wrapper.htm?arnumber=1041641.

Staroverov, Pavel, Silvia Marcos, Dimitry Kaynov, Mario Arbulú, Luis Cabas, and Carlos Balaguer. 2007. No Title. In Advances in Climbing and Walking Robots Proceedings of 10th International Conference CLAWAR 2007, ed. Ming Xie, Steven Dubowsky, Jean-Guy Fontaine, M Osman Tokhi, and Gurvinder S Virk, 591-598. Singapore. doi:10.1142/9789812770189_0068.

Staroverov, Pavel, Ricardo Martinez, Dimitry Kaynov, Mario Arbulú, Luis Cabas, and Carlos Balaguer. 2007. Detecting sound sources with the humanoid robot Rh-1. In Advances in Climbing and 
Walking Robots Proceedings of 10th International Conference CLAWAR 2007, ed. Ming Xie,

Steven Dubowsky, Jean-Guy Fontaine, M Osman Tokhi, and Gurvinder S Virk, 615-622. Singapore. doi:10.1142/9789812770189_0071. 